\title{
RECOBRIMENTO DE APATITAS "IN VITRO" SOBRE TITÂNIO - INFLUÊNCIA DO TRATAMENTO TÉRMICO
}

\author{
L. C. de O. VERCIK' ; C. M. de ASSIS ; M. V. LIA FOOK \\ M. L. dos SANTOS ; A. C. GUASTALDI ${ }^{l}$
}

Resumo

Titânio comercialmente puro, Ti-cp, e algumas de suas ligas são consideradas muito importantes na área médica devido sua excelente biocompatibilidade e propriedades mecânicas. Recentemente, foi desenvolvido um método químico, relativamente simples, para induzir a bioatividade desses materiais metálicos inertes, cujo princípio é imitar as condições biológica para obtenção do material desejado. Esta técnica denominada biomimético, foi utilizada para modificar a superfície do Ti-cp através do depósito de uma camada de apatita. $\mathrm{O}$ objetivo principal deste trabalho foi estudar a influência do tratamento térmico na evolução da cristalinidade das fases depositadas. Os recobrimentos de apatitas, com tratamentos térmicos entre 400 e $600^{\circ} \mathrm{C}$, mostraram através das técnicas de difração de raios-X e microscopia eletrônica de varredura, uma baixa cristalinidade semelhante às apatitas biológicas. Acima de $700^{\circ} \mathrm{C}$, os recobrimentos de apatita mostraram-se mais cristalinos apresentando uma mistura de fases de hidroxiapatita, fosfato octacálcico e fosfato de magnésio.

Palavras-chave: Biomateriais, titânio, recobrimento, processo biomimético, hidroxiapatita

\section{Introdução}

A diversidade das aplicações dos biomateriais, assim como o amplo espectro de sua composição química e a necessidade de formas e morfologias apropriadas para sua utilização, fazem da pesquisa nesta área do conhecimento um trabalho de característica eminentemente interdisciplinar, envolvendo fatores que definem o sucesso de suas aplicações, tais como: tratamento de superfície, rotas de sínteses, técnica de processamento (em formas variadas), degradabilidade e biocompatibilidade local e sistêmica. Desta forma, o desenvolvimento de biocerâmicas e de próteses deve ocorrer sob as mesmas condições de interdisciplinaridade que determinam o desenvolvimento de qualquer outro biomaterial [7].

Dentro deste contexto, a área de Química pode contribuir de forma significativa para a evolução desta pesquisa e para o aumento do leque de sua aplicabilidade, através do desenvolvimento de novos e eficazes biomateriais e também na elucidação dos mecanismos que governam a regeneração óssea e na compreensão básica de

1 Departamento de Fisico-Química - Instituto de Química - UNESP

CEP 14801-970 - Araraquara - SP - Brasil. 
algumas das características físicas e químicas das superfícies de vários metais, a partir dos quais se pode elevar ou estimular a nucleação de fosfato de cálcio in vitro. Como resultado, tem-se demonstrado que alguns materiais como o biovidro, hidroxiapatita - $\mathrm{Ca}_{10}\left(\mathrm{PO}_{4}\right)_{6}(\mathrm{OH})_{2}$ - sinterizada e alguns vidros cerâmicos, unem-se através de uma camada de apatita similar à do osso. Nestes materiais, tem-se estudado em tratamento químico de superfície, por exemplo, como a função dos radicais silanol (Si-OH) influenciam na formação e crescimento de uma camada de fosfato de cálcio em condições in vitro e in vivo [9].

O longo período de ligação interfacial entre um implante e o osso pode ser reduzido pela criação de uma superfície rugosa, ou de um filme de natureza porosa no implante, o que permite aumentar a área superficial disponível para a justaposição ossoimplante e aumentar a osseointegração [3], termo este definido como sendo o contato estabelecido entre o osso normal e remodelado com a superfície do implante, sem a interposição de tecidos conectivos que não sejam de células ósseas [6].

Cerâmicas a base de fosfato de cálcio estão sendo utilizados na odontologia e na medicina há mais de 30 anos. O grande interesse em uma fase particular dos tipos de fosfatos de cálcio, a hidroxiapatita (HA), surgiu devido sua grande similaridade com o principal componente presente na fase mineral do osso. A HA apresenta alta bioatividade (forte interação cerâmica/tecido ósseo) e biocompatibilidade, o que leva esse material ter um grande aceite como implante pelos tecidos vivos [1]. Uma das mais importantes aplicações da HA é como recobrimento de implantes metálicos, tais como titânio comercialmente puro, Ti-cp, e suas ligas. Esta idéia é de combinar as vantagens mecânicas dos metais com a excelente biocompatibilidade e bioatividade da HA.

Dentre as várias técnicas utilizadas para aplicação de recobrimentos de HA, temos as técnicas físicas $[12,13,19]$, as quais produzem recobrimentos de alta qualidade e filmes com alta densidade. As principais desvantagens dessas técnicas são a utilização de equipamentos sofisticados de alto custo e a baixa resistência à adesão devido à falta de uma ligação química entre o titânio e o recobrimento de apatitas. Esta é a principal razão pela qual novas técnicas, principalmente métodos químicos $[1,11,15]$, estão sendo estudadas para melhorar a adesão através da modificação de superfície do metal com diferentes tratamentos químicos, para que cristais de apatitas possam ser depositado quimicamente.

O método denominado biomimético e introduzido por Abe [1], é uma das técnicas mais promissoras para produção de biomateriais sob condições ambiente. Este método consiste na imersão do substrato, a ser recoberto, em uma solução sintética (SBF - Simulated Body Fluid) de composição química e pH semelhantes ao plasma sanguíneo e temperatura similar à do corpo humano. Devido a essas condições é possível recobrir materiais de formas complexas, como materiais porosos, e também materiais sensíveis a temperaturas, como é o caso dos polímeros. Além disso, com esta técnica pode-se recobrir implantes com diferentes fases de fosfatos de cálcio, as quais possuem características benéficas para formação óssea $[2,6]$.

\section{Materiais e métodos}

\section{Tratamento do Ti-cp com solução de $\mathrm{NaOH}$}

O substrato metálico (Ti-cp) utilizado para recobrimento de apatita foi cortado em pequenas barras com dimensão $0,5 \times 0,5 \times 0,3 \mathrm{~cm}$ e polidas com uma lixa 150 mesh. A Tabela 1, apresenta a análise química do Ti-cp utilizado, juntamente com as concentrações permitidas pela especificação da norma ASTM.

\begin{tabular}{|c|c|c|}
\hline Elementos & Ti-cp & ASTM F67-95b Ti-cp \\
\hline $\mathrm{O}$ & 0,0129 & 0,18 \\
\hline $\mathrm{N}$ & 0,0032 & 0,03 \\
\hline $\mathrm{C}$ & 0,0096 & 0,10 \\
\hline $\mathrm{H}$ & 0.005 & 0,015 \\
\hline $\mathrm{S}$ & 0,0005 & - \\
\hline $\mathrm{Ti}$ & balanço & balanço \\
\hline
\end{tabular}

Tabela 1. Composição química do Ti-cp $(\% \mathrm{~m} / \mathrm{m})$ Os substratos foram lavados em álcool, acetona e água deionizada durante 10 minutos no equipamento de ultra som. Em seguida, os substratos passaram por um tratamento em uma solução de $\mathrm{NaOH}(5 \mathrm{M})$ durante 24 horas a $60^{\circ} \mathrm{C}$.

Ecl. Quím., São Paulo, 28(1): 25-31, 2003 


\section{Recobrimento de apatita sobre o Ti-cp.}

Após o tratamento em solução de $\mathrm{NaOH}$, os substratos foram submetidos por 24 horas a $37^{\circ} \mathrm{C}$ em uma solução de silicato de sódio, $\mathrm{Na} 2 \mathrm{SiO} 3$, com posterior tratamento em uma solução de SBF (com concentração similar ao plasma sanguíneo) para uma nucleação mais lenta e organizada da apatita a ser depositada. Em seguida, os substratos foram imersos durante 6 dias a $37^{\circ} \mathrm{C}$ em uma solução 1,5 SBF para crescimento da mesma. A Tabela 2 apresenta as concentrações iônicas do plasma sanguíneo e de todas as soluções utilizadas neste processo.

\begin{tabular}{|c|c|c|c|c|c|c|c|c|c|}
\hline & $\mathrm{Na}^{+}$ & $\mathrm{K}^{*}$ & $\mathrm{Ca}^{2+}$ & $\mathrm{Mg}^{2+}$ & $\mathrm{LCO}_{3}{ }^{2}$ & $\mathrm{cr}$ & $\mathrm{IIPO}_{4}{ }^{2}$ & $\mathrm{SO}_{4}{ }^{20}$ & $\mathrm{SiO}_{3}{ }^{2}$ \\
\hline Plasma & 142,0 & 5,0 & 2,5 & 1,5 & 27,0 & 103,0 & 1,0 & 0,5 & - \\
\hline \multicolumn{10}{|c|}{ Sanguineo } \\
\hline SBF & 142,0 & 5,0 & 2,5 & 1,5 & 4,2 & $1+8,0$ & 1,0 & 0.5 & - \\
\hline 1,5 SBF & 213,0 & 7,5 & 3.8 & 2,3 & 6,3 & 223,0 & 1,5 & 0,75 & $\cdot$ \\
\hline $\mathrm{Na}_{4} \mathrm{SiO}_{3}$ & 2.0 & - & - & - & - & 1,0 & - & - & 3.6 \\
\hline
\end{tabular}

Tabela 2. Concentrações iônicas das soluções utilizadas para o recobrimento de apatitas $\left(\mathrm{mmol} . \mathrm{dm}^{-3}\right)$

\section{Tratamento térmico do recobrimento de apatita.}

Após o recobrimento os substratos foram submetidos a tratamentos térmico a 400, 500, 600, 700 e $800^{\circ} \mathrm{C}$, sem controle atmosférico e com taxa de aquecimento de $10^{\circ} \mathrm{C} / \mathrm{min}$.

\section{Caracterização do recobrimento de apatita.}

Todos os recobrimentos, após tratamentos térmicos, foram caracterizados utilizando Microscopia Eletrônica de Varredura acoplada a um analisador de espectroscopia por espalhamento de energia dispersiva de raios $\mathrm{X}$ e Difratometria de raios $\mathrm{X}$

\section{Resultados e discussões}

A superfície das amostras onde foi realizado o recobrimento de apatita foi polida utilizando uma lixa de granulometria 150 mesh, Figura 1a. Este tratamento foi realizado com o objetivo de aumentar a área específica e a energia superficial do substrato de Ti-cp [14,17].

Devido o metal de Ti-cp ser recoberto por uma fina camada passiva de óxido de titânio, $\mathrm{TiO} 2$, que se forma espontaneamente [8], este foi submetido a um tratamento utilizando uma solução de $\mathrm{NaOH} 5 \mathrm{M}$, com o intuito de produzir sobre a camada passiva de $\mathrm{TiO} 2$ uma superfície mais favorável e ativa para induzir a precipitação de apatita $[4,8,18]$, Figura $1 b$.

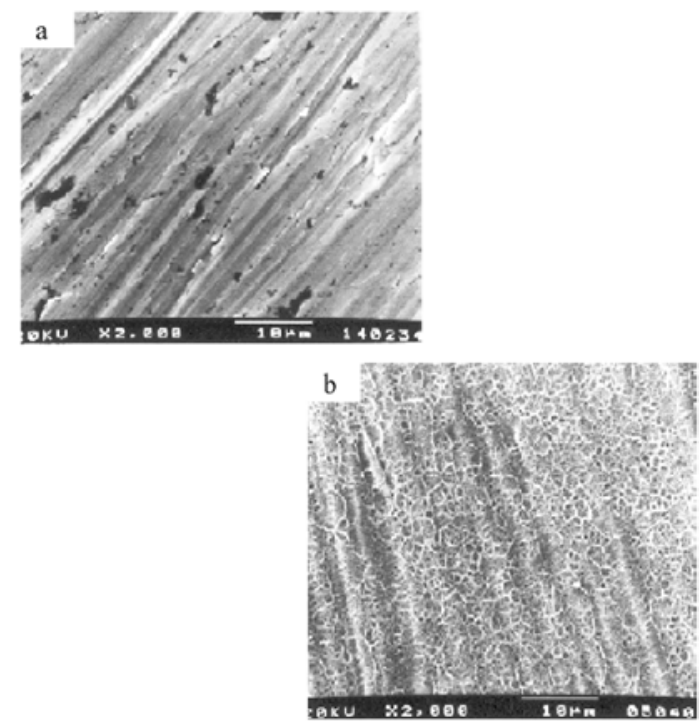

Figura 1. Microscopia Eletrônica de Varredura da superfície do Ti-cp (a) antes e (b) após o tratamento químico com uma solução de $\mathrm{NaOH}$.

Durante o tratamento alcalino a superficie passiva da camada de $\mathrm{TiO} 2$ pode reagir da seguinte forma $[5,8]$ :

$$
\begin{aligned}
& \mathrm{TiO}_{2}+\mathrm{OH}^{-} \quad \mathrm{HTiO}_{3}^{-} \\
& \mathrm{Ti}+3 \mathrm{OH}^{-} \quad \mathrm{Ti}(\mathrm{OH})_{3}^{+}+4 \mathrm{e}^{-} \\
& \mathrm{Ti}(\mathrm{OH})_{3}^{+}+\mathrm{e}^{-} \quad \mathrm{TiO}_{2} \mathrm{H}_{2} \mathrm{O}+1 / 2 \mathrm{H}_{2}(\mathrm{~g}) \\
& \mathrm{Ti}(\mathrm{OH})_{3}^{+}+\mathrm{OH}^{-} \quad \mathrm{Ti}(\mathrm{OH})_{4} \\
& \mathrm{TiO}_{2} n \mathrm{H}_{2} \mathrm{O}+\mathrm{OH}^{-} \quad \mathrm{HTiO}_{3}^{-} n \mathrm{H}_{2} \mathrm{O}
\end{aligned}
$$

Essas espécies carregadas negativamente combinam com os íons alcalinos na solução aquosa, resultando na formação de uma camada alcalina de hidrogel de titanato. Durante o tratamento térmico essa camada de hidrogel é desidratada e estabilizada como uma camada de titanato de sódio amorfo $[5,8]$.

A baixa capacidade de obtenção de grupos $\mathrm{Ti}-\mathrm{OH}$, decorrente do caráter anfótero do $\mathrm{TiO} 2$, faz com que os substratos de Ti-cp não formem núcleos 
suficientes para produzir uma camada densa e uniforme de apatitas. Portanto, com a imersão destes substratos em uma solução de um sal que apresente uma tendência a incrementar pontes de - $\mathrm{OH}$ e não desloque o equilíbrio do meio, possibilitará o aumento dos sítios ativos sobre a superfície do Ticp $[10,14]$. Neste trabalho foi utilizada uma solução de silicato de sódio, para aumentar a nucleação das apatitas, através da formação de grupos $\mathrm{Si}-\mathrm{OH}$, a qual é acelerada quando imersos em uma solução de SBF por 24 horas, tabela 2. Com a imersão dos substratos em uma solução 1,5 vez mais concentrada que a solução de SBF original, Tabela 2, os núcleos das apatitas crescem espontaneamente pelo consumo dos íons cálcio e fosfato da solução, formando uma camada uniforme de apatita

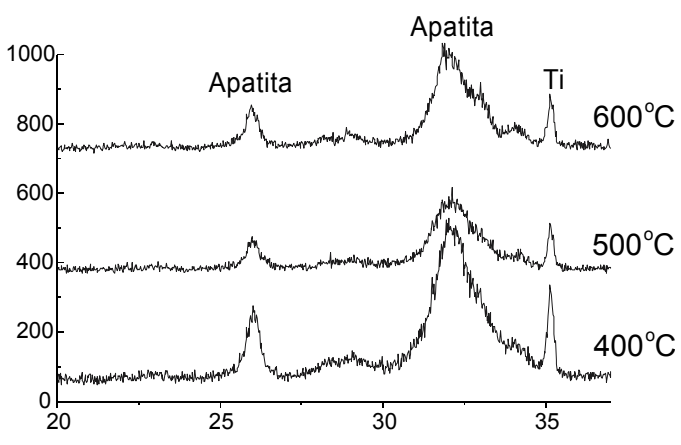

Figura 2. Difratometria de raios $X$ dos recobrimentos de apatita sobre a superfície do Ti-cp após tratamentos térmicos a 400,500 e $600{ }^{\circ} \mathrm{C}$.

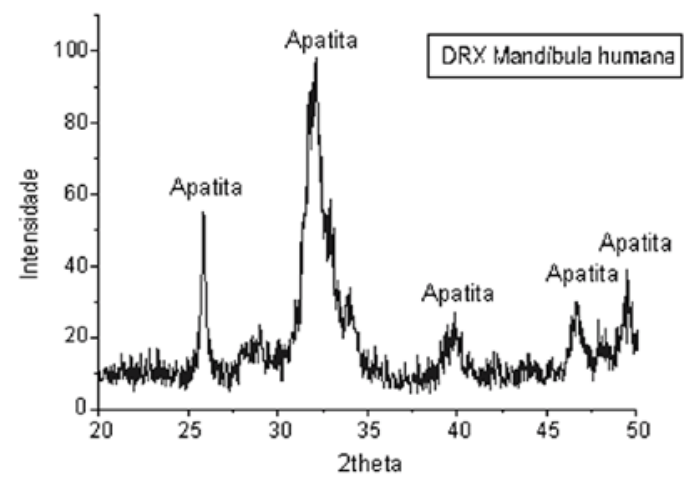

Figura 3. Difratometria de raios $\mathrm{X}$ apatita biológica: mandíbula humana.

A composição do recobrimento de apatitas sobre a superfície do hidrogel foi caracterizada por difratometria de raios X, Figuras 2 e 3. Observa-se que para os tratamentos térmicos a 400, 500 e 600 ${ }^{\circ} \mathrm{C}$, os difratogramas de raios $\mathrm{X}$ dos recobrimentos, Figura 2, apresentaram três bandas largas salientes localizadas a aproximadamente $2=26^{\circ}, 2=29^{\circ} \mathrm{e}$ $2=32^{\circ}$. Essas bandas são características do amplo número de picos referentes às fases apatitas, indicando uma estrutura pouco cristalina, bem similar à apatita biológica, Figura 3 . Os picos definidos em $2=27,5^{\circ}$ e $2=36^{\circ}$ são referentes ao Ti do substrato

A baixa cristalinidade das fases, no recobrimento, leva a sua instabilidade quando implantados. De acordo com a literatura $[5,6]$, fosfatos de cálcio amorfo e principalmente as fases fosfato tetracálcico e fosfato tricálcico possuem uma solubilidade bem superior a HA, levando o recobrimento a uma rápida desintegração e portanto à perda de fixação do implante, fenômeno conhecido como reabsorção

A Figura 4 mostra o aumento da cristalinidade do recobrimento de apatita com os tratamentos térmicos a 700 e $800^{\circ} \mathrm{C}$, sendo possível identificar alguns tipos de apatitas precipitadas sobre o substrato de Ti-cp. Foram observados juntamente com a fase cristalina da hidroxiapatita, $\mathrm{HA}$, picos referentes a fase fosfato octacálcico (OCP), $\mathrm{Ca}_{8} \mathrm{H}_{2}\left(\mathrm{PO}_{4}\right)_{6} \cdot{ }_{5} \mathrm{H}_{2} \mathrm{O}$ e fosfato de magnésio hidratado, $\mathrm{M}_{\mathrm{g} 3}\left(\mathrm{PO}_{4}\right)_{2} \cdot \mathrm{H}_{2} \mathrm{O}$. O surgimento destas fases cristalinas a partir de tratamentos térmicos superiores a $600{ }^{\circ} \mathrm{C}$ indica uma inflexão da temperatura de transição vítrea $(\mathrm{Tg})$ dos diversos tipos de apatitas presentes. Isto indica que a temperatura de cristalização do OCP e do fosfato de magnésio são superiores a $600^{\circ} \mathrm{C}$.

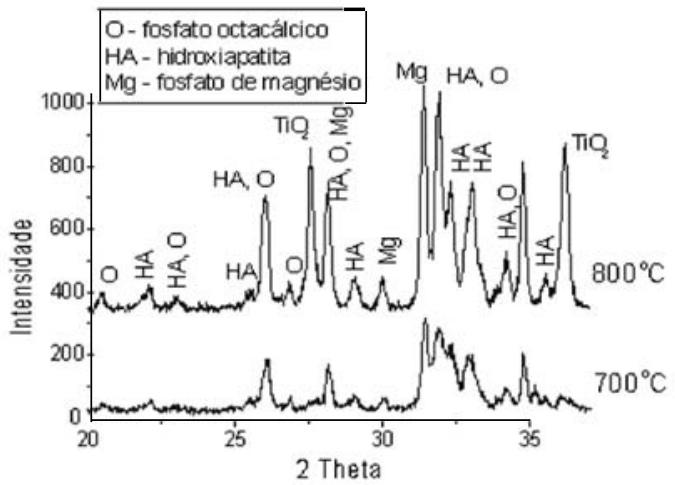

Figura 4. Difratometria de raios $\mathrm{X}$ dos recobrimentos de apatita sobre a superfície do Ti-cp após tratamentos térmicos a 700 e $800{ }^{\circ} \mathrm{C}$. 
A fase OCP é considerada muito promissora devido a sua participação na formação óssea, e também por ser precursora da fase HA. Segundo Feng [4], para se conseguir recobrimentos de fosfatos de cálcio sobre superfícies de Ti é necessário que a solução esteja acima do valor de equilíbrio de saturação do OCP. Uma vez que a velocidade de crescimento de OCP excede sua velocidade de hidrólise, existe a probabilidade de formação de uma mistura de fases de OCP e HA [16].

As fotos de microscopia eletrônica de varredura do recobrimento de apatitas sobre Ticp, Figura 5 e 6, mostraram um depósito com morfologia uniforme, composto por partículas esféricas com tamanho entre 3 e $5 \mu \mathrm{m}$. Pode-se observar também alguns aglomerados devidos à fusão de algumas partículas após os tratamentos térmicos.
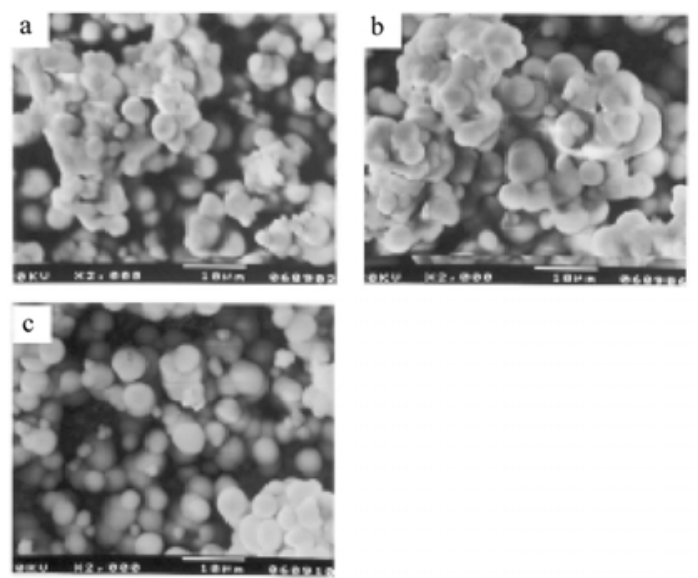

Figura 5. Microscopia Eletrônica de Varredura dos recobrimentos de apatita sobre a superfície do Ti-cp após tratamentos térmicos a (a) 400 (b) 500 e (c) $700{ }^{\circ} \mathrm{C}$.

Com o aumento da temperatura de tratamento foi possível observar uma morfologia similar para todas as temperaturas. Todos os recobrimentos apresentaram-se isentos de trincas, o que é muito comum de se encontrar quando estes são submetidos a tratamentos térmicos. Nas fotos de MEV, Figuras $6 \mathrm{a}$ e $6 \mathrm{~b}$, com tratamentos térmicos a 700 e $800{ }^{\circ} \mathrm{C}$ comprovou-se o aparecimento de uma outra fase, também detectada pelo DRX. Esta fase com uma morfologia de aspecto esponjosa foi caracterizada como sendo a fase $\mathrm{Mg}_{3}\left(\mathrm{PO}_{4}\right)_{2} \cdot \mathrm{H}_{2} \mathrm{O}$, confirmada também através da análise de EDS, com o aparecimento do elemento magnésio, Figura 6c.
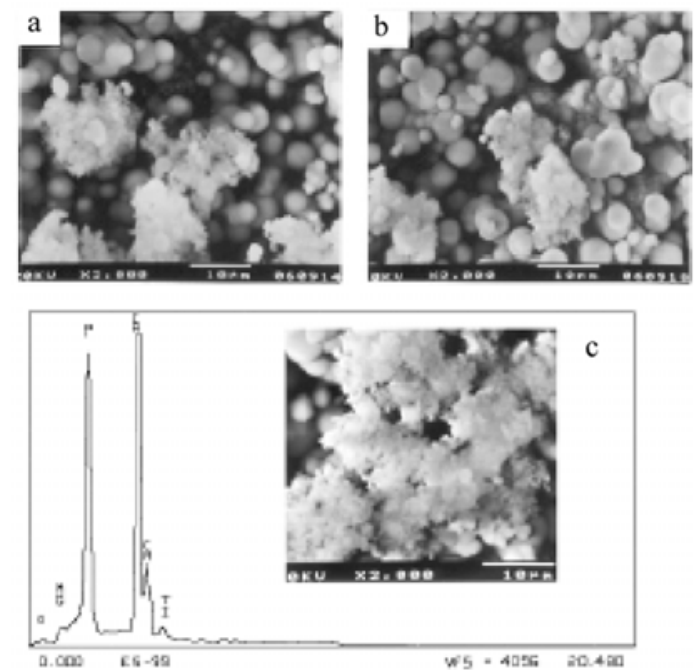

Figura 6. Microscopia Eletrônica de Varredura dos recobrimentos de apatita sobre a superfície do Ti-cp após tratamentos térmicos a (a) 700 , (b) $800{ }^{\circ} \mathrm{C}$ e (c) EDS da partícula esponjosa.

Segundo alguns autores e trabalhos anteriores [2,8,17], a utilização direta de uma solução de SBF mais concentrada tem uma grande influência na quantidade e na composição dos fosfatos precipitados. Assim sendo, a imersão dos substratos de Ti-cp em uma solução de SBF por 24 horas a $37^{\circ} \mathrm{C}$ faz com que o processo de nucleação de apatita seja mais lento, proporcionando uma formação mais ordenada dos núcleos e favorecendo uma maior quantidade da fase HA.

\section{Conclusões}

Os resultados obtidos utilizando-se o processo biomimético em duas etapas, proporcionaram um recobrimento com uma camada de apatitas uniforme, homogênea e com uma boa porosidade. Com o processo de nucleação lento, a fase majoritária encontrada foi a hidroxiapatita. As outras fases presentes apareceram em pequenas quantidades, sendo a fase fosfato octacálcico um precursor da fase hidroxiapatita. Com tratamentos térmicos entre 400 e $600^{\circ} \mathrm{C}$, as fases apresentaramse pouco cristalinas, com uma evolução a temperaturas superiores a $700{ }^{\circ} \mathrm{C}$. Este processo de recobrimento de apatitas mostrou-se extremamente eficiente para obtenção da fase 
hidroxiapatita semelhante à biológica, com baixa cristalinidade mesmo quando submetidas a tratamentos térmicos entre 400 e $600^{\circ} \mathrm{C}$

\section{AGRADECIMENTOS}

À Fapesp (processo 00/11125-9), CNPq/ PIBIC e CAPES/PICDT.

VERCIK, L. C. de O.; ASSIS, C. M. de; LIA FOOK, M. V.; SANTOS, M. L. dos; GUASTALDI, A. C. Apatites coating "in vitro" on titanium - influence of the thermal treatment.

\begin{abstract}
Commercially pure titanium (cp-Ti), and some of its alloys are considered very important in the medical field because of its excellent biocompatibility and mechanical properties. Recently a simple chemical method was introduced to induce the bioactivity of these inert metallic materials. In this work, the biomimetic chemical process has been used to modify the cp-Ti surface with the deposit of a layer of apatite. The main objective consisted in studying the influence of the thermal treatment on the evolution of the crystallinity of the deposited phases. X-ray diffraction and scanning electron microscopy techniques showed that the apatite coatings with thermal treatments between 400 and $600{ }^{\circ} \mathrm{C}$ presented low crystallinity similar to the biological apatites. Above $700{ }^{\circ} \mathrm{C}$, the apatite coatings appeared more crystalline, exhibiting a mixture of hydroxyapatite, octacalcium phosphate and magnesium phosphate.
\end{abstract}

Keywords: Biomaterials, titanium, coatings, biomimetic process, hydroxyapatite.

\section{Referências bibliográficas}

[1] ABE, Y.; KOKUBO, T.; YAMAMURO, T. Apatite coatings on ceramics, metals and polymers utilising a biological process. J. Mater. Sci.: Mater. Med., v. 1, p. 536$540,1990$.

[2] BARRERE, F; LAYROLLE, P.; VAN BLITTERSWIJK, C. A.; DE GROOT, K. Biomimetic calcium phosphate coatings on Ti6Al4V: A crystal growth study of octacalcium phosphate and inhibition by $\mathrm{Mg} 2+$ and $\mathrm{HCO} 3-$. Bone, v. 25 , n. 2, p. $107 \mathrm{~s}-111 \mathrm{~s}, 1999$

[3] BROWNE, M.; GREGSON, P. J. Effect of mechanical surface pre-treatment on metal ion release. Biomaterials, v. 21, p. $385-392,2001$.

[4] FENG, Q. L.; CUI, F. Z.; WANG, H.; KIM, T. N.; KIM, J. O. Influence of solution conditions on deposition of calcium phosphate on titanium by $\mathrm{NaOH}$-treatment. $J$. Crystal Growth, v. 210, p. 735-740, 2000.

[5] GIL, F. J.; PADRÓS, A.; MANERO, J. M.; APARÍCIO, C.; NILSSON, M.; PLANELL, J. A. Growth of bioactive surfaces on titanium and its alloys for orthopedic and dental implants. Mater. Sci. Eng., v. 22, p. 53-60, 2002.

[6] GROSS, K. A.; BERNDT, C. C.; GOLDSCHLAG, D. D.; IACONO, V. In vitro changes of hydroxyapatite coatings. Quintessence, v. 12, p. 589-597, 1997.

[7] KAWACHI, E. Y.; BERTRAN, C. A.; DOS REIS, R. R.; ALVES, O. L. Biocerâmicas: tendências e perspectivas de uma área interdisciplinar. Quím. Nova, v. 23, p. 518-522, 2000 .

[8] KOKUBO, T. Apatite formation on surfaces of ceramics, metals and polymers in body environment. Acta Mater., v. 46, n. 7, p. 2519-2527, 1998.

[9] MARTINEZ-PÉREZ, C. A.; MARTINEZVILLAFANE, A.; ROMERO-GARCIA, J. Formación de hidroxiapatita sobre una superficie polimérica por un método biomético. Rev. Mex. Ing. Biom., v. 21, p. 137-141, 2000.

[10] MIYAJI, F.; KIM, H.; HANDA, S.; KOKUBO, T.; NAKAMURA, T. Bonelike apatite coating on organic polymers: novel nucleation process using sodium silicate solution. Biomaterials, v. 20, p. 913-919, 1999.

[11] MONMA, H. Electrochemical deposition of calciumdeficient apatite on stainless steel substrate. J. Ceram. Soc. Jap., v. 101, n. 7, p. 578-583, 1993.

[12] NIE, X.; LEYLAND, A.; MATTHEWS, A. Deposition of layered bioceramic hydroxyapatite/TiO2 coatings on titanium alloys using a hybrid technique of micro-arc oxidation and electrophoresis. Surf. Coat. Techn., v. 125, p. $407-414,2000$.

[13] PARK, E.; CONDRATE SR, R. A. Graded coating of hydroxyapatite and titanium by atmospheric plasma spraying. Mater. Lett., v. 40, p. 228-234, 1999.

[14] RIGO, E. C. S. Recobrimento biomimético de implantes metálicos com hidroxiapatita. São Carlos, 2001. 146f. Tese (Doutorado) - Departamento de Ciências e Engenharia de Materiais, Universidade Federal de São de Carlos, São Carlos, 2001.

[15] RIGO, E. C. S.; OLIVEIRA, L. C.; SANTOS, L. A.; Ecl. Quím., São Paulo, 28(1): 25-31, 2003 
CARRODÉGUAS, R. G.; BOSCHI, A. O. Implantes metálicos recobertos com hidroxiapatita. Rev. Bras. Eng. Biom., v. 15, n. 1-2, p. 21-29, 1999.

[16] SHI, J.; DING, C.; WU, Y. Biomimetic apatite layers on plasma-sprayed titanium coatings after surface modification. Surf. Coat. Techn., v. 137, p. 97-103, 2001.

[17] VERCIK, L. C. O.; ALENCAR, A. C.; RAMIREZ, I.; GUASTALDI, A. C. Dental implants: surface modification of cp-Ti using plasma spraying and the deposition of hydroxyapatite. Mater. Sci. Forum: Adv. Powder Techn. III, v. 418, p. 669-673, 2003.

[18] WEN, H. B.; DE WIJN, J. R.; LIU, Q.; DE GROOT, K. A simple method to prepare calcium phosphate coatings on Ti6Al4V. J. Mater. Sci.: Mater. Med., v. 8, p. 765-770, 1997.

[19] ZENG, H.; LACEFELD, W. R. XPS, EDX and FTIR analysis of pulsed laser deposited calcium phosphate bioceramic coatings: the effects of various process parameters. Biomaterials, v. 21, p. 23-30, 2000. 